\section{A safe approach to perform endoscopic mucosal resection of a duodenal adenocarcinoma located close to a duodenal diverticulum}
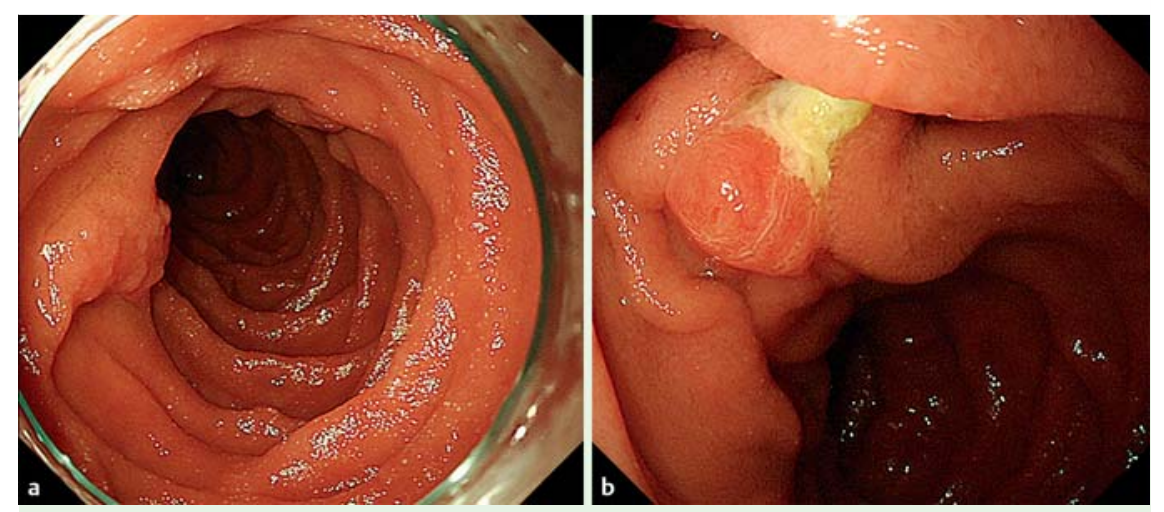

Fig. 1 Endoscopic views in an 83-year-old woman showing: a a polypoid duodenal lesion located close to; $\mathbf{b}$ a duodenal diverticulum that was proximal to the papilla of Vater.

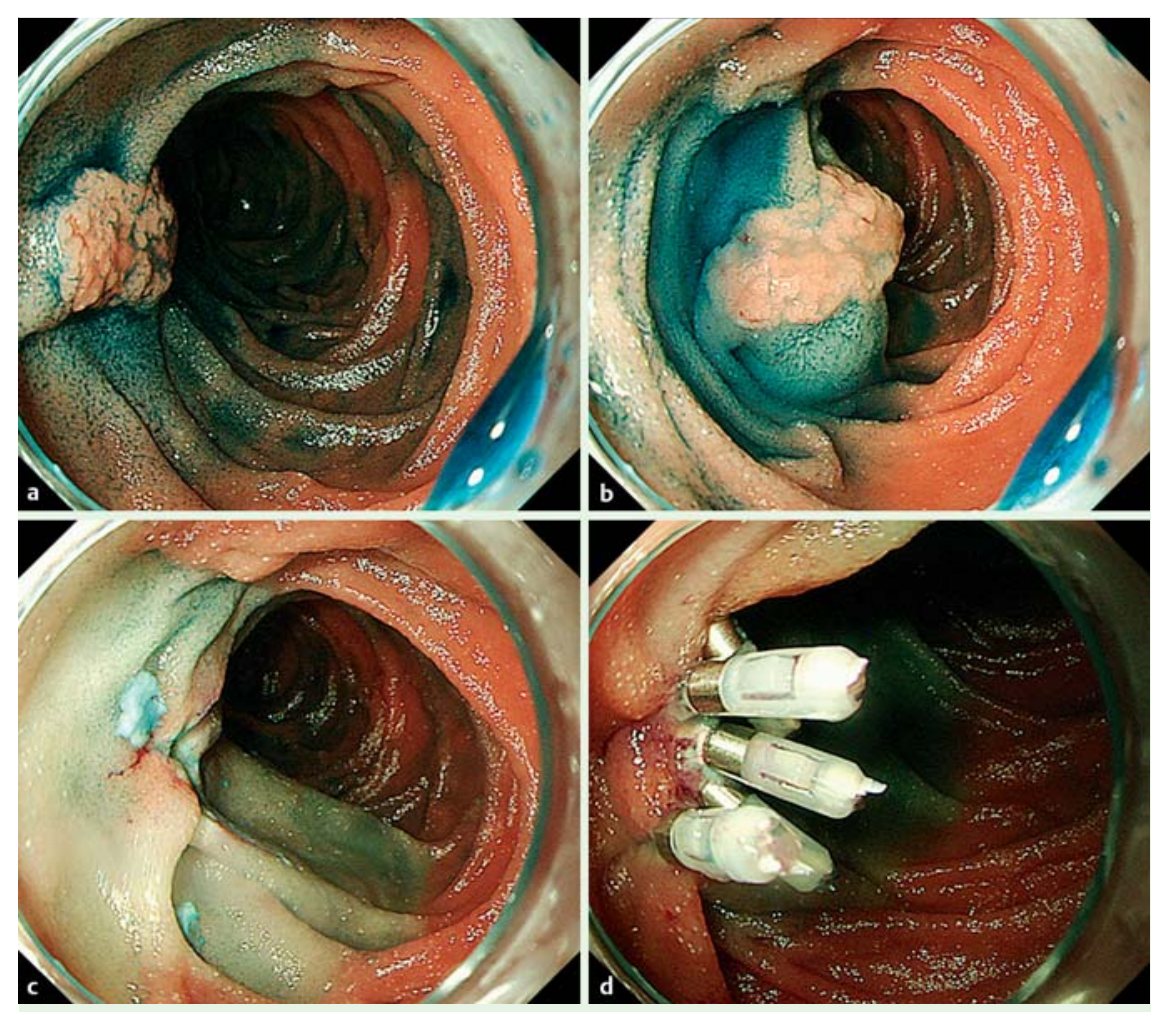

Fig. 2 Views during chromoendoscopy in the duodenum using $0.2 \%$ indigo carmine solution showing: a the margin of the tumor clearly delineated; $\mathbf{b}$ the submucosal cushion that was created by injecting a mixture of indigo carmine and saline into the base of the lesion; $\mathbf{c}$ an endoscopic appearance that is consistent with complete resection (R0); $\mathbf{d}$ the hemoclips that were placed to close the mucosal defect.

Not infrequently duodenal adenomas and adenocarcinomas are located in difficult or awkward positions that can impede their resection endoscopically. Duodenal diverticuli are common, occurring in 5\% of individuals. Herein, we describe a safe technique for the removal of an early duo- adenocarcinoma. The patient's preference was to undergo an endoscopic excision. The following steps that allowed endoscopic resection to be safely performed were followed ( Video 1 ). (i) The margin of the tumor was delineated by chromoendoscopy using $0.2 \%$ indigo carmine solution ( Fig. 2a). (ii) An indigo carmine and saline mixture was injected into the base of the lesion to create a submucosal cushion. Submucosal injection into the distal side of the tumor pushed the lesion away from the diverticulum and resulted in it being positioned en face to the endoscope ( Fig. 2 b). (iii) The tip of a snare (SnareMaster; Olympus Medical, Tokyo, Japan) was used to precut the distal side of the lesion. Freeing up of the tissue by precutting allowed the tip of the snare to be anchored and stabilized, which subsequently allowed the lesion to be more readily ensnared. (iv) The snare was then placed circumferentially around the tumor; and ( $\mathrm{v}$ ) the lesion was resected in one piece ( Fig.2c). (vi) Complete closure of the defect was accomplished with hemoclips (Olympus Medical) ( $\bullet$ Fig. 2d). The patient's postoperative course was uneventful. She started a soft diet 1 day after the procedure and was discharged home 5 days later. Histology of the resected specimen revealed a $13-\mathrm{mm}$, intramucosal, well-differentiated tubular adenocarcinoma, without evidence of lymphovascular invasion.

Endoscopic resection techniques have now become accepted therapies for the removal of duodenal neoplasms [1-3]. However, complications are much more common than when resecting stomach or colorectal lesions, especially with endoscopic submucosal dissection $[1,4,5]$, because the duodenum has a very thin wall and the mucosal defect is exposed to the digestive forces of bile and pancreatic juice. Therefore, a careful approach, such as described in this case, is mandatory.

\section{Video 1}

A video showing the steps necessary to safely perform duodenal endoscopic resection. A polypoid duodenal lesion was found proximal to a duodenal diverticulum near to the papilla of Vater. Superficial chromoendoscopy with indigo carmine was used to delineate the tumor margins and a submucosal cushion was created. The distal side of the lesion was precut using the tip of a snare. This allowed the snare to be anchored and stabilized, before it was placed circumferentially around the tumor. The lesion was resected in one piece and the defect was closed completely with hemoclips. 
Endoscopy_UCTN_Code_TTT_1AQ_2AD

Competing interests: None

Seiichiro Abe, Ichiro Oda, Hiroyuki Takamaru, Satoru Nonaka, Haruhisa Suzuki, Shigetaka Yoshianaga, Yutaka Saito

Endoscopy Division, National Cancer Center Hospital, Tokyo, Japan

\section{References}

1 Nonaka S, Oda I, Tada $K$ et al. Clinical outcomes of endoscopic resections for nonampullary duodenal tumors. Endoscopy 2014. In press

2 Matsumoto S, Yoshida Y. Selection of appropriate endoscopic therapies for duodenal tumors: an open-label study, single-center experience. World J Gastroenterol 2014; 20: $8624-8630$

3 Yamamoto Y, Yoshizawa N, Tomida $\mathrm{H}$ et al. Therapeutic outcomes of endoscopic resection for superficial non-ampullary duodenal tumor. Dig Endosc 2014; 26 (Suppl. 02): $50-56$

4 Hoteya S, Yahagi N, Iizuka T et al. Endoscopic submucosal dissection for nonampullary large superficial adenocarcinoma/adenoma of the duodenum: feasibility and long-term outcomes. Endoscopy International Open 2013; 1: E2-E7

5 Inoue T, Uedo N, Yamashina T et al. Delayed perforation: a hazardous complication of endoscopic resection for non-ampullary duodenal neoplasm. Dig Endosc 2014; 26: $220-227$
Bibliography

DOI http://dx.doi.org/

10.1055/s-0034-1390917

Endoscopy 2014; 46: E676-E677

(c) Georg Thieme Verlag KG

Stuttgart · New York

ISSN 0013-726X

\section{Corresponding author}

Seiichiro Abe, MD

Endoscopy Division

National Cancer Center Hospital

5-1-1 Tsukiji, Chuo-ku

Tokyo 104-0045

Japan

Fax: +81-3-3542-3815

seabe@ncc.go.jp 